\title{
Primary-Simultane Operation Pri Politrauma
}

\author{
Shaposhnikov Veniamin Ivanovich* \\ Noncommercial educational private institution of higher education Kuban Medical Institute Head. Chair of morphologic disciplines. \\ Professor of surgical diseases, Vice Rector, Krasnodar, Russia
}

*Corresponding author: Shaposhnikov Veniamin Ivanovich, Noncommercial educational private institution of higher education Kuban Medical Institute Head. Chair of morphologic disciplines. Professor of surgical diseases, Vice Rector, Krasnodar, Russia

\begin{abstract}
The author examines the current problems of modern medicine associated with the growth of injuries. In recent decades, not only has the number of injuries increased, but so does the severity of the damage. There were many problems, both in the organization of treatment of victims, and in the methods of surgical correction of injuries, especially if they are combined - multiple, and at the same time injured up to a dozen or even more persons. This is most often the case in road traffic accidents, and they sometimes occur on sections of highways, away from the regional and regional centers, where the main medical staff are concentrated, and in rural areas, where hospitals operate with very limited medical staff. For example, the staffing of the CRB usually provides surgical and trauma departments for 40-60 beds, that is, assistance is planned. or a surgeon, or a traumatologists, but often come persons with trauma of those organs and systems that are to be treated by a neurosurgeon, angiosurgeon and other specialists. The difficulty lies not only in the absence of them, but also in the tools for such operations. One doctor of the CRB receives a load, which in the regional center is allowed 5-10 or more specialists of surgical profile. In this situation, doctors of rural hospitals should have the technique to perform surgery in any form of trauma, but for this they must pass the school of training in leading surgical centers, and now they are mostly former students who know everything, but nothing they know how to do it. Admittedly, the increase in fatalities from injuries is partly due to this factor. The author gives an instructive observation of polytrauma, which fits into the context of the problem at hand.
\end{abstract}

Keywords: Polytrauma; Surgical treatment; Treatment

\section{The Aim of the Study}

To show the importance of the surgeon's training in providing emergency care to victims of polytrauma

\section{Introduction}

Currently, there is a widespread increase in injuries, which is due to the rapid mechanization of work and recreation of the person. With the increase in injuries, there were many problems in the organization of treatment of victims with different types of injuries. The role of peripheral treatment facilities (mainly CRB) in providing effective assistance to traumatized persons has also increased, taking into account the mass migration of the population during the warmer months from cities to villages [1]. The structure of injuries has also changed significantly - the number of combined, multiple and combined multiple injuries is increasing. At the same time, the combined injury includes damage to different systems, and to multiple - the same [2]. With the one-time increase in the number of injured organs and systems, attitudes on surgical aggression have changed, and this has been greatly facilitated by recent advances in anesthesiology, allowing the most severe and pro-longed operations [3,4]. Although until very recently it was thought that expanding the volume of surgery on the human body is dangerous for his life $[5,6]$. However, the healing process of victims with polytrauma, dictates the need not only to own, but also to perform complex simulative manipulations on various organs and systems. In primary osteosynthesis performed in a combined injury, poor treatment results are twice as common as in other methods of cross-section, and the consolidation of the fracture occurs on average 1 month faster [7-9]. X-ray diagnosis is also needed to clarify the nature and type of injury, but it increases the radial effects on humans [10]. For this reason, it is necessary to resort to magnetic resonance imaging (MRI), as well as to be able to perform ancillary manipulations, including laparocenteza, in case of suspected abdominal injuries [11,12]. However, the leading role in the diagnosis of all injuries still belongs to clinical methods of examination, and they future doctors master during their studies at the university. On this basis, it is necessary to strengthen control over the quality of training of future health professionals in order to eliminate the appearance of ballast, which only interferes with 
the goal - reducing fatality from injuries, if in reducing their number they are powerless, for it is a national problem.

\section{Material and Methods}

In one of the district hospitals of Krasnodar region observed 59 victims with combined-multiple trauma, of which 41 (69\%) were affected. primary simulates were performed (from 2 to 6). The rest had 18 (31\%) Victims and this was due to their extremely severe condition, or because of the fracture of the bones of the base of the skull, or because of a closed heart injury, limited only to conservative methods of treatment - skeletal stretching, skeletal hanging of the lower limb. All of these patients died five to 18 hours after hospitalization. All patients (41) who had surgery, had multiple fractures of tubular bones, 32 - traumatic brain injury, 6 - closed rupture of abdominal organs, 3 - a fracture of the spine, 3 - pelvic bones, 12 - ribs, 10 - bones. A total of 204 organs and tissues were injured, ranging from 2 to 8 in one person. At the time of hospitalization, everyone had a second- to third-degree shock. The patients were between 13 and 52 years old. The diagnosis was based on clinical, X-ray, endoscopic, instrumental and laboratory examination. All of these patients had metal osteosynthesis of tubular bones (from 2 to 5, only 86), 4 - removed spleen, 2 stitches on the liver, 6 - trepanation of the skull, etc. Of the 41 victims, 19 had simulated operations carried out 2-6 hours after hospitalization, and the rest within 1 day. The duration of surgical aggression ranged from 2 to 6 hours. Operations were performed under general end tracheal anesthesia with the use of controlled breathing hardware. Two traumatologists took part in the surgical intervention with or without the involvement of a surgeon. The 23-year-old man was admitted after a road accident in a state of extreme severity - pulse filamentous, AD - is not determined, breathing superficial 6-8 in 1 minute. On examination - on the face a lot of abrasions, a parting squint, pupils slightly enlarged and sluggishly react to light, in the left temporal area of the hematoma, from the left ear canal released a blood-bracing liquor (on the $\mathrm{X}$-ray revealed a fracture of the temporal bones). The victim has a closed fracture of both forearms and lower third of the left thigh, and an open middle third of both shins and the middle third of the right thigh. The man was intoxicated. The trachea intubation was carried out and superficial anesthesia was carried out against the background of hardware breathing. After 6-8 hours, the blood pressure rose to $100 / 50 \mathrm{~mm}$ h. pillar, but soon began to fall, and the stomach to increase in volume. Performed laparocenteza, which revealed blood in the abdominal cavity. Suspected two-moment rupture of the spleen and on vital signs performed laparotomy, which confirmed the diagnosis. A splenectomy was performed, and simultaneous operations were performed on the bone and joint system with the involvement of two more hospital traumatologists, i.e. two teams of specialists worked simultaneously. Sustained metorosemetalostheism of both tibia bones and on Rush of both femurs was performed. The simultaneous surgical creativity lasted about 6 hours, with 5 operations performed. Closed reposition of fragments of both forearms with plaster bandage. The general superficial anesthesia lasted about 2 days, and all this time the hardware breath was carried out. The hospital treatment lasted almost 3 months. There were no complications after the surgery. Gradually, all the fractures consolidated. However, he could not walk without crutches because of pain in his left hip joint. The resulting radiation load did not allow X-rays of this joint, and it was sent to the regional center for MRI. Magnetic resonance imaging unexpectedly revealed an old medial fracture of the cervix of the hip, which was the cause of pain and dysfunction of this joint. All previously diagnosed fractures have been consolidated. He refused hip neck surgery. For 1 year he was disabled in the 2 nd group. Further communication with him was lost.

\section{The Result}

All the victims safely underwent surgery, but 2 (4.8\%) after 3-5 days developed a fat embolism of the cerebral vessels, from which they died. Thus, out of 59 patients with polytrauma, only 20 (33.9\%) died. Thirty-nine survivors had no early post-operative surgery. The duration of inpatient treatment in them on average was 45 days (36 to 90 days). The duration of incapacity in 5 patients was 6 months, 21 - 8 months, 10 - more than a year. 3 (7.7\%) patients had a persistent disability - 2 it was caused by the consequences of a severe traumatic brain injury, and 1 - a false joint of the cervix of the hip.

\section{Discussion}

Thus, the performance of primary Simultane operations in combined-multiple trauma is practically and economically justified activity. They make it possible to successfully combat shock by creating stability of fragments and early activation of victims. The very method of operation, which allows to use existing metal structures effectively, is of great importance in the outcome of the operation. The materials presented in the article fully confirm the data of the literature that these operations reduce the number of postoperative complications by 2 times; on average for 1 month accelerate the consolidation of breaks, which accelerates the rehabilitation of victims. The cause of failure sits largely depends on the severity of the brain and heart injury, and this problem is now waiting to be solved.

\section{Conclusion}

Primary simultane operations in combined-multiple trauma can be attributed to the achievements of modern medicine. They allow doctors in polytrauma to actively influence the correction of homeostasis disorders and accelerate the consolidation of bone fractures, which has a clear economic effect. The introduction of new surgical technologies will lead to further progress in the treatment of polytrauma.

\section{References}

1. Shaposhnikov VI (2007) Injury Prevention. Kuban's printed yard Krasnodar, p. 34.

2. Yumashev GS (1977) Traumatologists and Orthopedics, pp. 503.

3. Cousin MI (2015) Surgical Diseases. Textbook, GEOTAR - MEDIA. 
4. De Siqueira J, Tawfiq O, Garner J (2014) Managing the open abdomen in a district general hospital. Ann R Coil Surg Engl 96(3): 194-198.

5. Burich MP (1999) General Foundations of surgical technology, pp. 445.

6. Krasnov AF, Arshin VM, Ceitlin MD (1984) Directory on Traumatology, pp. 397.

7. Belyakov AA, Capitansky IS, Sergeev VM (1988) Tactics for the treatment of multiple bone fractures in combination with traumatic brain injury. Proceedings V Congress of Orthopaedic Traumatologists 1: 65-67.

8. Becishor VK, Kozub KN, Kodryanu KD (1988) One-stage osteosynthesis of patients with multiple fractures and their consequences. Works $\mathrm{v}$ Congress of Orthopaedic Traumatologists 1: 69.
9. Lebedev AA, Shlakov VV (1988) Intra-bone metal osteosynthesis in the treatment of combined and multiple di-aphysary fractures of the hip and shins. Proceedings V Congress of Traumatologists-orthopedics of the USSR 1: 111 - 112.

10. Kiszkovsky AN, Tyutin LA (1989) Emergency X-ray diagnostics, pp. 304.

11. Chernousov AF (2012) Surgical Diseases. Textbook. GEOTOR - MEDIA, pp. 525.

12. Shaposhnikov VI (1986) Initial simultaneous operations for combined and multiple fractures of long tubular bones, Surgery 11: 19-21.
To Submit Your Article Click Here:

This work is licensed under Creative

Commons Attribution 4.0 License

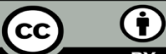

DOI: $10.32474 /$ SCSOAJ.2019.03.000163

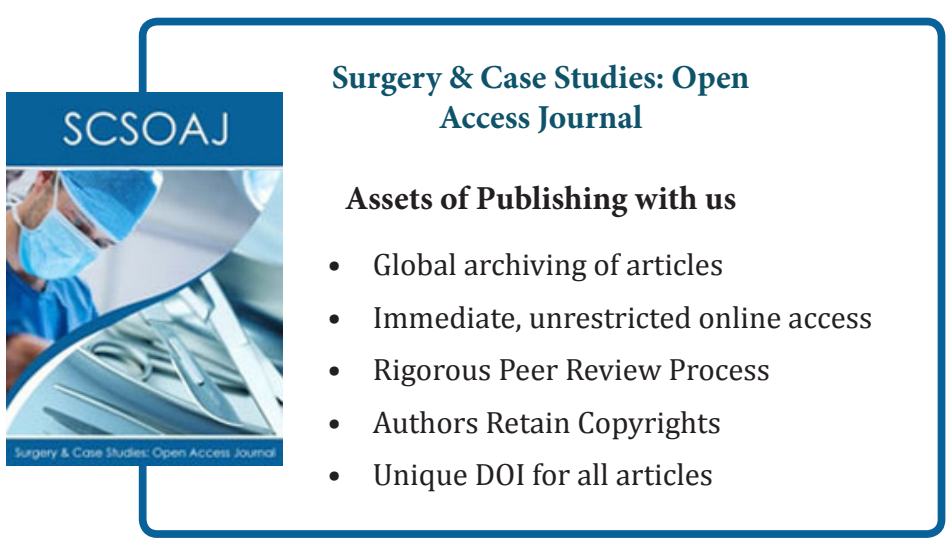

